\title{
Multi-Voltage Level Active Distribution Network with Large Share of Weather-Dependent Generation
}

This paper was downloaded from TechRxiv (https://www.techrxiv.org).

\section{LICENSE}

CC BY 4.0

SUBMISSION DATE / POSTED DATE

01-10-2021 / 07-10-2021

\section{CITATION}

Baviskar, Aeishwarya; Das, Kaushik; Koivisto, Matti Juhani; Hansen, Anca Daniela (2021): Multi-Voltage Level Active Distribution Network with Large Share of Weather-Dependent Generation. TechRxiv. Preprint. https://doi.org/10.36227/techrxiv.16722355.v1

$\mathrm{DOI}$

10.36227/techrxiv.16722355.v1 


\title{
Multi-Voltage Level Active Distribution Network with Large Share of Weather-Dependent Generation
}

\author{
Aeishwarya Baviskar, Student Member, IEEE, Kaushik Das, Senior Member, IEEE, \\ Matti Koivisto, Member, IEEE, and Anca Daniela Hansen, Member, IEEE
}

\begin{abstract}
Utility-scale and small scale wind and solar power installations along with electric vehicle charging stations, and other active sources of energy are increasing at the medium and lower voltage levels in the distribution grid. This situation required a better understanding of the impact of high penetration of weather-dependent renewable energy sources on the operating conditions of the distribution network at both medium and low voltage levels. Despite the need, a multi-voltage level distribution network model, based on real network data and weather-dependent renewable generation data, has not been presented for distribution grid studies. This paper presents a comprehensive multi-voltage level active distribution network model based on real network data along with load and generation time-series for about a year. The network topology is modelled based on geographical data for various rural, semi-urban, and urban locations. The distribution network is embodied with a large share of renewable generation sources, with generation time-series simulated from meteorological data. The network is also flexible to incorporate other assets such as electric vehicle charging stations, storage, etc. Thus, the presented active distribution network model can be used to study, optimize, and control the effects of weather-dependent generation and other network assets in the distribution grid.
\end{abstract}

Index Terms-active distribution network, load profile, generation profile, network topology, renewable generation, network assets

\section{INTRODUCTION}

D ISTRIBUTED Renewable Energy Sources (RES), including utility-scale installations and small-scale projects closer to the point of consumption, have a major role to fulfill the ambitious renewable energy targets set by various countries. Distributed RES are also crucial in providing access to energy in developing and under-developed economies around the world. Feed-in tariff programs and financial support schemes were key drivers to increase the share of renewable energy sources in the grid $[1,2,3]$. In recent years, declining electricity cost via renewable energy sources and growing interest in self-consumption have boosted the distributed renewable energy markets in Europe as well as the world [4]. Weather-dependent energy sources, like solar photovoltaics (PV) and wind power plants (WPP), are at the forefront, accounting for a large share in utility-scale, commercial, and user-end installations [4]. Furthermore, storage units, electric vehicle (EV) charging stations, combined heat power plants are gaining increasing popularity, and have the potential to become an integral part of the future distribution grid. Thus, a future distribution network resembles more closely an active network

Aeishwarya Baviskar, Kaushik Das, Matti Koivisto, and Anca Hansen are with Technical University of Denmark with multiple assets such as weather-dependent generation, combined heat cycle plants, electric vehicle charging, etc. There is, however, a growing concern among distribution system operators (DSOs) for loss of revenue, increased operation cost, and the threat of disintermediation [6, 7].

The main hurdle behind the cost-effective operation of distribution network with a large share of weather-dependent RES is due to their intermittent/fluctuating and non-dispatchable nature as discussed in [1]-[3]. However, power electronics present in RES such as PVs and WPPs, electricity storage units, EV charging stations, imparts these installations with advanced control capabilities which convert them into active network assets. Additionally, these assets are connected at different voltage levels (owing to the power carrying capabilities of different voltage levels). For example, in distribution networks, some of WPPs are connected at $60 \mathrm{kV}$ and $10 \mathrm{kV}$ nodes, while the commercial or roof-top PVs are connected at $10 \mathrm{kV}$ and $0.4 \mathrm{kV}$ nodes. When the assets are controlled in each of these different voltage levels, the change in power flows not only impact that specific voltage level but also other voltage levels too in terms of voltage profile, power losses, reactive power flow, etc. This has a further impact on the active and reactive power flow to the transmission network. It is thus necessary to study, analyze, and develop active management techniques for the growing number of beneficial network assets connected in the distribution grid. As a result, a benchmark distribution network model representing the characteristics of a large share of integrated generations in each voltage level is of particular interest.

The design of benchmark models in power systems has been a topic of interest for many research works. A brief summary of some network models is presented in Table. I The benchmark models presented by the CIGRE Task force are one the most commonly used and well known distribution network benchmark models. The CIGRE Task Force developed distinct high, medium and low voltage benchmark networks for studying and analyzing network behavior in presence of distributed energy sources including RES [4]-[6]. However, the network topology for the CIGRE models does not represent a real distribution network. Another set of benchmark models that demand noteworthy attention are the IEEE distribution network models [7]. The IEEE distribution feeders were developed with multiple objectives such as testing new algorithms or distribution system elements such as transformers in unbalanced networks. The IEEE distribution feeders do not focus on high share of renewable generation. In addition, only 24 hours of load time-series data is available with the IEEE distribution 
feeders and most of the networks are synthetic, except for the 8500 IEEE distribution feeder. GridLAB-D is an open source power systems simulation environment with inbuilt transmission and distribution network models also based on synthetic network topology with taxonomic load time-series data for different scenarios such as a hot summer day, or a cold winter day [8] developed especially to study quasi steady state operations and smart-grid applications. Furthermore, to address the overestimates of grid extension needed due to increasing distributed energy sources and storage installations in transmission and distribution networks, synthetic open-source medium voltage distribution networks, called the $e$ Go grid models, are presented in [9]. The eGo grid models are designed using an open source software called the DIstribution Network GeneratOR (DINGO) for generating synthetic networks, emphasizing the growing interest in analyzing distribution network behavior pertaining to the aforementioned developments. Sarstedt et. al. [10] also presents an integrated transmission and distribution network model, wherein the topology for the lower voltage networks is synthetically generated. Some

TABLE I

SUMMARY OF A FEW OPEN-SOURCE DISTRIBUTION NETWORK MODELS

\begin{tabular}{|c|c|c|c|c|}
\hline & Application & $\begin{array}{l}\text { Voltage } \\
\text { level }\end{array}$ & $\begin{array}{l}\text { Network } \\
\text { Topology }\end{array}$ & Time-series \\
\hline [4] & $\begin{array}{l}\text { Power Flow } \\
\text { analysis }\end{array}$ & Distribution; & Synthetic & - \\
\hline [11] & $\begin{array}{l}\text { Congestion } \\
\text { management, } \\
\text { transmission } \\
\text { capacity, grid } \\
\text { stability, etc. }\end{array}$ & Transmission & $\begin{array}{l}\text { German: from } \\
\text { geographical } \\
\text { data }\end{array}$ & - \\
\hline [12] & $\begin{array}{l}\text { open source } \\
\text { planning } \\
\text { platform }\end{array}$ & $\begin{array}{l}\text { Distribution; } \\
\text { unbalanced }\end{array}$ & Synthetic & $\begin{array}{l}1 \text { hour resolu- } \\
\text { tion for } 1 \text { year }\end{array}$ \\
\hline [8] & $\begin{array}{l}\text { Quasi- } \\
\text { steady state } \\
\text { time-series } \\
\text { solutions, } \\
\text { smart grid } \\
\text { studies }\end{array}$ & All & $\begin{array}{l}\text { USA; } \\
\text { Synthetic }\end{array}$ & $\begin{array}{l}\text { Taxonomy } \\
\text { models } \\
\text { representing } \\
\text { different days }\end{array}$ \\
\hline [13] & $\begin{array}{l}\text { Power Flow } \\
\text { analysis }\end{array}$ & $\begin{array}{l}\text { Transmission } \\
\text { and Distri- } \\
\text { bution }\end{array}$ & $\begin{array}{l}\text { German; } \\
\text { Partly } \\
\text { synthetic }\end{array}$ & $\begin{array}{l}15 \text { min res- } \\
\text { olution for } 1 \\
\text { year }\end{array}$ \\
\hline [7] & $\begin{array}{l}\text { Testing new } \\
\text { algorithms, } \\
\text { and } \\
\text { distribution } \\
\text { system } \\
\text { elements }\end{array}$ & $\begin{array}{l}\text { Distribution; } \\
\text { unbalanced }\end{array}$ & $\begin{array}{ll}\text { USA } & \& \\
\text { Europe: } & \\
\text { Synthetic } & \end{array}$ & $\begin{array}{l}\text { One minute } \\
\text { resolution for } \\
24 \text { hours }\end{array}$ \\
\hline
\end{tabular}

network models have been developed recently with the help of open source geographical data such as Open Street Maps [14] which represents a real distribution network more closely than synthetic models. Works addressing generation or synthesis of benchmark models can be found in [9], [15], [16]. SciGRID is an example of open source network model intended towards studying congestion management, transmission capacity, grid stability in the transmission networks developed on data from Open Street Maps [11]. To enhance understanding of network behavior and characteristics, and needs of future distribution networks with RES and other low-carbon technologies, [17] establishes Low Voltage Network Models and Low Carbon Technology Profiles founded on the UK distribution network.
SimBench is one of the more recent additions to the library of open-source benchmark models developed on principles presented in [13], [16]. The grid data in SimBench is in accordance with the German Distribution System Operator (DSO)'s operation and planning principle with partly synthetic network topology. Furthermore, SimBench also provides load time-series for about one year for different types of consumer behavior.

More often the network topology in the open-source models is based on synthetic network modeling. Amongst all the distribution network models presented so far, there are two multi-voltage network models which include the low voltage levels [10], [13] and only [12], [13] is accompanied with load time-series data for over a year. However, to study the effect of weather-dependent generation in a future active distribution grid, it is crucial to also study the correlation between the load demand and weather-dependent generation at different voltage levels in the network. Thus, a real distribution grid model with focus on load time-series and correlated weather dependent generation time-series, and the ability to incorporate with modern network assets such as storage, EV charging, combined heat plants, etc. is lacking. In this respect, this research proposes and presents such a multivoltage level distribution network model, which is needed for an exhaustive study of the performance, operation and control of future weather-dependent distribution networks in terms of grid requirements and specifications, flexibility requirements for future RES technologies. The presented model can also be used in developing advanced control strategies to improve the overall operating conditions of such distribution networks while incorporating additional network assets.

The proposed distribution network model, is an open source network model, entitled as the DTU 7k Bus Active Distribution Network (DTU-ADN), is accompanied by load time-series aggregated at $60 \mathrm{kV}, 10 \mathrm{kV}$ and $0.4 \mathrm{kV}$ derived from measurement data and correlated weather-dependent generation time-series simulated from meteorological data. The network topology for the DTU-ADN is generated from geographical data and represents real distribution networks. Furthermore, it has the flexibility to incorporate additional network assets to investigate performance of active distribution network with combined heat and power plants, storage, wide spread use of electric vehicles, etc. The objective behind the development of the DTU-ADN is the development of control and optimization algorithms for active management of future distribution network with aforementioned assets.

Some novel features of the proposed and developed benchmark distribution network model are as following:

- Weather-dependent generation time-series simulated from meteorological data and correlated load time-series, derived from measurement data at three different voltage levels

- Capability to co-simulate a multi-voltage distribution network with large share of RES at different voltage levels to evaluate impact of distributed generation on the operational conditions of a distribution network

- 18 different realistic and diverse network topology of $10 \mathrm{kV}-0.4 \mathrm{kV}$ networks with different characteristic loads, 
such as agricultural, household, and/or industrial, and distributed RES

- Flexibility to incorporate additional network assets such as combined heat and power plants, storage units, electric vehicle charging stations, etc.

- Comprehensive platform for development of coordinated control between different assets for optimal operation of distribution network, while incorporating uncertainty from weather dependent generation and loads.

Section II briefly introduces the DTU $7 k$ Bus Active Distribution Network along with the publicly available data for the network. The raw data used to develop the distribution network model and methodology is described in section III. Results of the implemented methodology are presented in section IV, and section $\mathrm{V}$ summarizes the presented active distribution grid model and the research methodology by highlighting its crux.

\section{DTU 7K-Bus ACTIVE Distribution Network}

The DTU 7k-Bus Active Distribution Network (DTU-ADN) [18] is a balanced three-phase multi-voltage network with a high share of weather-dependent renewable energy generation. The complete dataset for DTU-ADN is open-source. It spans across three voltage levels, $60 \mathrm{kV}-10 \mathrm{kV}-0.4 \mathrm{kV}$, while being connected to the transmission grid via step-up $60 \mathrm{kV} / 150 \mathrm{kV}$ transformers. The medium voltage (MV) $60 \mathrm{kV}$ network can be connected to 17 distinct $10 \mathrm{kV}-0.4 \mathrm{kV}$ networks, simultaneously or in combinations, at different $60 \mathrm{kV}-10 \mathrm{kV}$ substations. This section presents key features of the DTU 7k-Bus Active Distribution Network.

\section{A. The $60 \mathrm{kV}$ Network}

The topology of the $60 \mathrm{kV}$ network originates from a real Danish grid. It hosts 25 buses out of which 23 buses connect $60 \mathrm{kV} / 10 \mathrm{kV}$ substations, and one bus connects to the transmission grid via $60 \mathrm{kV}-150 \mathrm{kV}$ step-up transformers. Onload tap changers are present at the $60 \mathrm{kV}-150 \mathrm{kV}$ transformer and also at the $60 \mathrm{kV}-10 \mathrm{kV}$ substation transformers. The $60 \mathrm{kV}$ network hosts three WPPs with installed capacities of $12 \mathrm{MW}$, $15 M W$, and $15 M W$ composed of type IV controllable wind turbines. Fig. 1 illustrates the network topology with its key elements.

Measurement data in a real distribution network, based on historical data for 1 year, forms the basis of the load (active and reactive power) and generation time series accompanying the $60 \mathrm{kV}$ grid. Aggregated load time series at all the $60 \mathrm{kV} / 10 \mathrm{kV}$ substations, and generation time series for the three WPPs are provided for the $60 \mathrm{kV}$ network. Due to the presence of distributed generation at $10 \mathrm{kV}-0.4 \mathrm{kV}$ voltage levels, there is a significant reverse power flow in the network. Negative active power values in the aggregated load time series denote reverse power flow. As a consequence of a large share of distributed generation, negative values, or reverse power flow is also observed in the aggregated load time series at the $60 \mathrm{kV}-150 \mathrm{kV}$ substation. In addition, 17 different $60 \mathrm{kV}-10 \mathrm{kV}$ substations can be expanded with $10 \mathrm{kV}-0.4 \mathrm{kV}$ networks to form the complete three voltage level network.

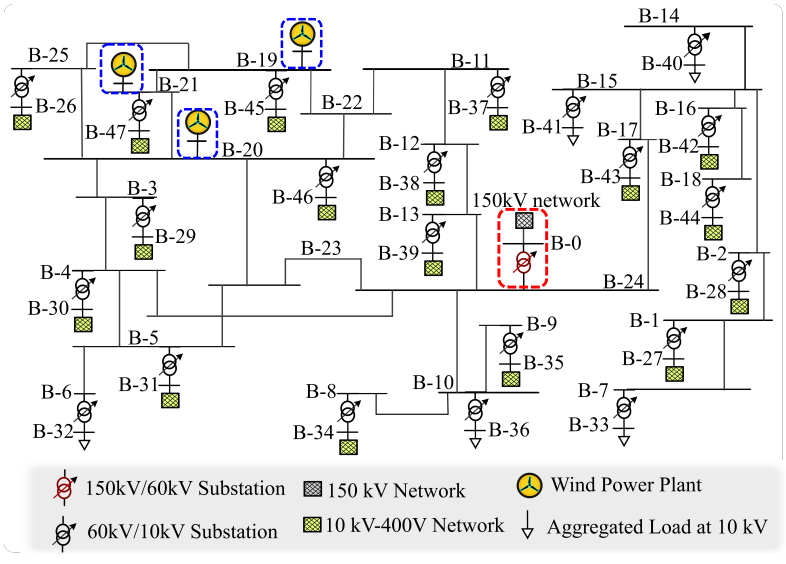

Fig. 1. Network topology for the $60 \mathrm{kV}$ network in the DTU 7k-Bus Active Distribution Network indicating locations of the Wind Power Plants, and $10 \mathrm{kV}-0.4 \mathrm{kV}$ networks

\section{B. $10 \mathrm{kV}-0.4 \mathrm{kV}$ Networks}

Topology for the $10 \mathrm{kV}-0.4 \mathrm{kV}$ networks associated with the DTU-ADN represents unique layouts as they are derived from publicly available data from open street maps [14] using Distribution Network Models module (DiNeMo) [19]. The networks cover varied geographical areas, thus making some networks dense, akin to urban networks, and others more sparse, comparable to rural networks. The number of supply or generation nodes in the networks falls in the range of 40 to 650 nodes. Cumulatively, the entire DTU-ADN consists of approximately 6541 nodes at $0.4 \mathrm{kV}, 427$ nodes at $10 \mathrm{kV}$, and 25 nodes at $60 \mathrm{kV}$ which accounts for its name DTU 7kBus Active Distribution Network. In addition, there are a total of $29110 \mathrm{kV} / 0.4 \mathrm{kV}$ substations with off-load tap changing transformers within the $10 \mathrm{kV}-0.4 \mathrm{kV}$ networks. Table II and III list statistics for the $10 \mathrm{kV}-0.4 \mathrm{kV}$ networks at Bus 27 and Bus 46 respectively.

The DTU-ADN dataset also specifies unique load profiles for each of the $10 \mathrm{kV}-0.4 \mathrm{kV}$ nodes. Since, the DTU-ADN is a balanced three-phase distribution network model, the $10 \mathrm{kV}$, and $0.4 \mathrm{kV}$ nodes serve multiple customers connected to these nodes, thus the load and generation time series mirror aggregated values. The load time series provided with the dataset are categorized into four main categories, namely household, industrial including commercial, agricultural, and miscellaneous loads. In total there are 27 different load time series derived from [16]. All the $10 \mathrm{kV}-0.4 \mathrm{kV}$ networks have varied proportions of load profiles from each category. Thus, the $10 \mathrm{kV}-0.4 \mathrm{kV}$ networks can be further classified in terms of their cumulative maximum load demand from each category into predominantly residential networks, highly industrialized networks, or agricultural networks.

\section{Weather-Dependent Generation}

Numerous nodes in the $10 \mathrm{kV}-0.4 \mathrm{kV}$ networks have either solar or wind or both generation sources installed along with the load. The assortment of installed solar capacities is from a minimum of a few $k W s$ to approximately $2.5 M W$. Similarly, installed wind capacities range from a minimum of $300 \mathrm{~kW}$ 
to a maximum of $16 M W$. The cumulative installed solar and wind capacities in the DTU-ADN are approximately $26 \mathrm{MW}$ and $150 M W$ respectively.

There are approximately 1900 distributed generators across the $10 \mathrm{kV}-0.4 \mathrm{kV}$ networks, amongst which about 300 installations across 17 networks can actively participate in the grid operations via advanced power electronics control. The distinction between passive and actively participating distributed generation is made according to the Danish grid codes and may vary depending on the local grid codes. The presence of smaller distributed generation units $(<11 k W)$ along with the controllable units account for their different effects on distribution grid operations due to their uncontrollable and controllable properties.

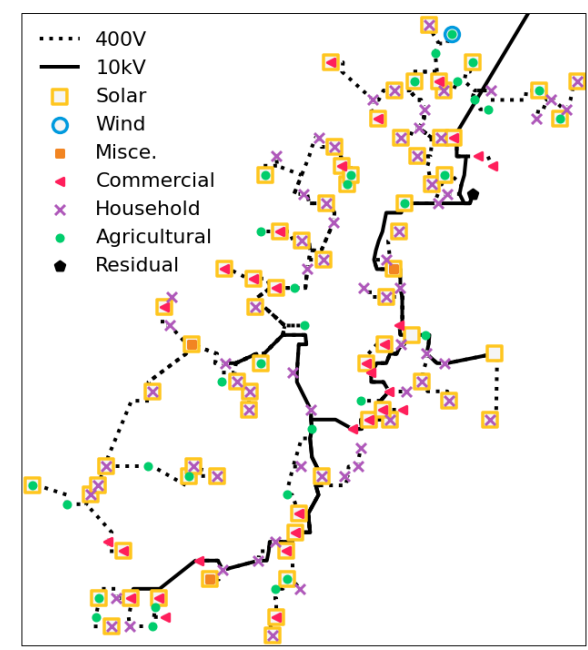

Fig. 2. The $10 \mathrm{kV}-400 \mathrm{~V}$ network at Bus 27 illustrating $10 \mathrm{kV}$ and $0.4 \mathrm{kV}$ distribution lines, type of load profiles at each node, location of solar and wind power installations

The network image for the $10 \mathrm{kV}-0.4 \mathrm{kV}$ network at bus 27 is given in Fig. 2 for exemplification. Network at bus 27 is chosen for visualization because it is one of the smaller networks, in terms of the number of nodes, and also hosts all four categories of loads. Fig. 2 indicates the network topology, with the distribution lines, all the nodes with the load profile category, and type of generation installed. The cumulative capacity of solar generation in this network is $1.85 \mathrm{MW}$ which is distributed amongst numerous nodes, and the wind capacity is $0.44 M W$ at a single node. RES installed in the network accounts for $34 \%$ of the cumulative maximum load demand from the network and supplies approximately $13 \%$ of the total energy need in the given time frame.

\section{Possible Applications}

The DTU-ADN serves as a multi-voltage distribution network model with diverse distribution network topology, at different stages of renewable energy penetration, simulated based on geographical data. The DTU-ADN data set can be used to analyze the effect of a high share of renewable energy in rural, semi-urban, or urban network topology. It can extended to analyze the diurnal, seasonal, weather and temperature dependent effects on the active and reactive power demand, voltage profiles, power losses, etc. from a distribution network using the time-series data provided with the network dataset. Furthermore, effect on distribution network assets, such as on-load tap changers, voltage regulators, switching capacitors, can be studied in presence of a highly weatherdependent power flow.

The DTU-ADN can be used as a platform to study various control strategies at medium or low voltage levels and analyze their effects on the overall performance of the network with correlated load and weather-dependent generation. It provides a unique opportunity to investigate the long term effects of actively controlling large number of distributed renewable generation in local network operations of a distribution network as well as the interaction between transmission and distribution networks. In addition, the network model is flexible to incorporate storage, electric vehicles, combined heat plants, hybrid power plants etc. at the distribution level. This enhances and expands its applicability to multiple study areas.

\section{E. How to access the data?}

The DTU-ADN is an open-source dataset available in an online repository [18]. The dataset in the repository is the first version of the DTU 7k-Bus Active Distribution Network and is subject to updates as the work progresses.

All the network files in the dataset are csv files suitable to be directly used in MatPower, PandaPower or Pypower. Further description of the data can be found in the readme file included.

\section{NETWORK MODELING METHODOLOGY}

This section describes the methodology used to generate the DTU 7k-Bus Active Distribution Network (DTU-ADN). The first sub-section presents the raw data used in DTU-ADN model generation. The second sub-section outlines an optimization algorithm, which decomposes the aggregated $60 \mathrm{kV}$ load-generation profile into individual $10 \mathrm{kV}$ or $0.4 \mathrm{kV}$ loadgeneration profiles. The final sub-section describes a heuristic algorithm used to assign load-generation profiles to individual $10 \mathrm{kV}$ or $0.4 \mathrm{kV}$ nodes.

\section{A. Raw Data}

$60 \mathrm{kV}$ Network: The network topology, line, and transformer data for the $60 \mathrm{kV}$ network is based on a real but anonymous Danish distribution network. The aggregated load timeseries at $10 \mathrm{kV}$ and the WPP generation data is derived from measurement data from this network. It should be noted that there have been major restructuring in this specific network; however, the network model and data are good representatives of a real network. This data forms the basis on which the entire DTU 7k-Bus Active Distribution Network is developed.

$10 \mathrm{kV}$ Network Topology: The network topology for all the $10 \mathrm{kV}-0.4 \mathrm{kV}$ networks are generated from an open-source online tool, DiNeMo, provided by the European Commission, Joint Research Centre [19]. DiNeMo takes as input an area of interest from Open Street Map [14] and reproduces a 
representative distribution grid. The inputs to DiNeMo for generating $10 \mathrm{kV}-0.4 \mathrm{kV}$ networks in this work are based on an approximate geographical area for the location of the $60 \mathrm{kV} / 10 \mathrm{kV}$ substations in Fig. 1. In addition to the geographical location of the distribution network DiNeMo also takes as input, population / consumer density, probability of consumers per building [\%], Minimum low voltage demand $[\mathrm{kW}$, Minimum MV demand [kW], Voltage level $[\mathrm{kV}]$, and transformer capacity for the location. The National Denmark statistical data provides inputs to generate realistic $10 \mathrm{kV}$ $0.4 \mathrm{kV}$ networks in terms of energy consumption patterns and load distribution. Thus, the DTU-ADN represents a set of realistic and diverse networks in terms load distribution, consumption and network topology as highlighted through the example networks presented in Table II and III.

The $10 \mathrm{kV}-0.4 \mathrm{kV}$ network generated by DiNeMo includes distribution line data, $\mathrm{MV} / \mathrm{LV}$ substation data, switches in the network, and approximate value of the load at each node. The load data from DiNeMo lists a demand in $k V A$ for each node based on the maximum demand input provided at the time of network generation. The demand in $k V A$ provided by DiNeMo is then used to scale and assign Standard Load Profile (SLP)s at every node in the network.

Standard Load Profiles: The SLPs provided by SimBench [20] are used in this work to derive load time-series for individual $0.4 \mathrm{kV}$ and $10 \mathrm{kV}$ nodes.

Wind and Photovoltaic generation profiles: The wind power generation profiles and photovoltaic profiles provided with the data set are generated using the DTU Correlations in Renewable Energy Sources (CorRES) simulation tool using meteorological time-series and stochastic simulations [21].

Geographical location of the PV plant, installed capacity [MW], surface azimuth angle and surface tilt are the input to CorRES to generate PV generation profiles for the given time. Global Solar Atlas provides inputs for the surface azimuth angle and tilt for the chosen geographical location [22]. Since, the geographical span of the $10 \mathrm{kV}-0.4 \mathrm{kV}$ networks is constrained to a small area, a uniform PV output is assumed throughout each $10 \mathrm{kV}-0.4 \mathrm{kV}$ network and only one PV profile is generated per $10 \mathrm{kV}-0.4 \mathrm{kV}$ network. The PV generation profile output from CorRES is in per unit.

The data register for WPPs provides a comprehensive list of WPP installations across Denmark along with their hub heights, installed capacities, number of turbines, turbine rated power, turbine diameter, etc. [23] The input data for simulating WPP's generation profile in CorRES is thus directly taken from the data register [23]. WPPs located geographically closer to the $60 \mathrm{kV} / 10 \mathrm{kV}$ substation are chosen for simulation. Thus, there may be multiple WPP generation profile associated with a $10 \mathrm{kV} / 0.4 \mathrm{kV}$ network. Even though CorRES inputs the installed capacity of a WPP, the output WPP generation profile is in per unit.

\section{B. Optimization Algorithm}

The optimization aims to break down the load time-series aggregated at $10 \mathrm{kV}$ into a weighted sum of SLPs and weatherdependent generation profiles as shown in Fig. 3. The residual time-series in Fig. 3 refers to a characteristic component of the load time-series aggregated at $10 \mathrm{kV}$ which cannot be encapsulated via individual or combination of any available SLP and generation time-series. The optimization is performed individually for each of the $10 \mathrm{kV}-0.4 \mathrm{kV}$ networks.

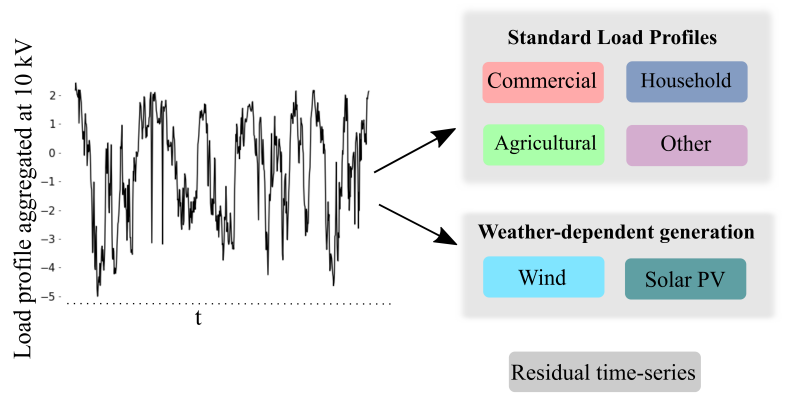

Fig. 3. Breakdown for load time-series aggregated at $10 \mathrm{kV}$ into weighted sum of standard load profiles, weather-dependent generation, and the residual time-series

Let $\mathcal{L}$ be the set of all available SLPs and $\mathcal{W}$ be the set of all weather-dependent generation profiles available for one $10 \mathrm{kV}-0.4 \mathrm{kV}$ network. $\mathcal{R}$ is the set of real numbers and $\mathcal{Z}$ is the set of integers. The following notations are defined for the optimization algorithm,

- $P_{t}$ : load aggregated at $10 \mathrm{kV}$, at time $\mathrm{t}$ in $M W$

- $\mathbf{L}_{t}$ : vector containing load from all SLPs at time $t$ in $p . u$.

- $l_{i, t}$ : power demand for the $i^{t h}$ SLP at time $t$ in p.u., where $i \in \mathcal{L}$

- $\mathbf{W}_{t}$ : vector with weather dependent generation in p.u.

- $w_{j, t}$ : generation from the $j^{t h}$ generation profile at time $t$ in p.u., where $j \in \mathcal{W}$

- D: vector containing maximum load from all the SLPs in $M W$

- $d_{i}$ : maximum load for the $i^{\text {th }}$ SLP in $M W$, where $i \in \mathcal{L}$

- $\mathbf{G}$ vector containing installed generation capacity for all available generation profiles for a particular $10 \mathrm{kV}-0.4 \mathrm{kV}$ network in $M W$

- $g_{j}$ : installed generation capacity from the $j^{\text {th }}$ generation profile in $M W, j \in \mathcal{W}$

The data flow in the optimization algorithm and dynamic time warping process is depicted in Fig. 4. Load time-series aggregated at $10 \mathrm{kV}\left(P_{t}\right)$, SLP load vector $\left(\mathbf{L}_{t}\right)$, and weatherdependent generation vector $\mathbf{W}_{t}$ are the inputs to the optimization. The outputs from the optimization are the maximum load vector for each SLPs (D), and a vector containing installed generation capacity of PV and wind in the network $(\mathbf{G})$. The dynamic time warping process is explained in the following section.

$\mathbf{L}_{t}$, a vector of load for all SLPs, in p.u., at time $t$, can be written as,

$$
\mathbf{L}_{t}=\left[\begin{array}{llll}
l_{1, t} & l_{2, t} & \ldots & l_{27, t}
\end{array}\right]
$$

$\mathbf{W}_{t}$, a vector with weather-dependent generation, in $p . u$., at time $t$, can be written as

$$
\mathbf{W}_{t}=\left[\begin{array}{llll}
w_{1, t} & w_{2, t} & \ldots & w_{k, t}
\end{array}\right], k \in \mathcal{Z}
$$




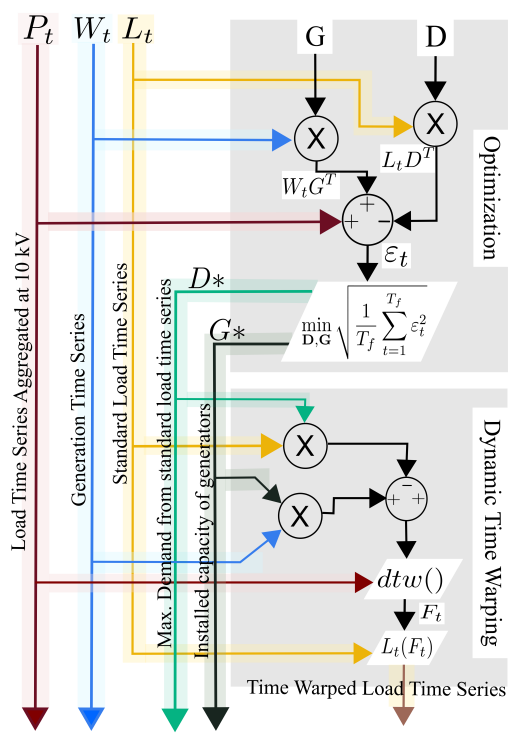

Fig. 4. Flowchart depicting the input and output data from the optimization algorithm and dynamic time warping process

Vector with cumulative maximum demand for all SLPs, D, can be written as,

$$
\mathbf{D}=\left[\begin{array}{llll}
d_{1} & d_{2} & \ldots & d_{27}
\end{array}\right]
$$

and vector $\mathbf{G}$ containing the maximum weather-dependent generation for solar and wind profiles, is written as,

$$
\mathbf{G}=\left[\begin{array}{llll}
g_{1} & g_{2} & \ldots & g_{k}
\end{array}\right], k \in \mathcal{Z}
$$

Let $\varepsilon_{t}$ denote the difference between load profile aggregated at $10 \mathrm{kV}$ and weighted sum of SLPs and weather-dependent generation, at time $t$, representing the residual time-series,

$$
\varepsilon_{t}=P_{t}+\mathbf{W}_{t} \mathbf{G}^{T}-\mathbf{L}_{t} \mathbf{D}^{T}
$$

It is assumed that all the $10 \mathrm{kV}-0.4 \mathrm{kV}$ networks have local generation sources. The local generation in the network is accounted by adding the weighted generation time series, $\mathbf{W}_{t} \mathbf{G}^{T}$, to the load time-series aggregated at $10 \mathrm{kV}, P_{t}$. Thus, weighted sum of the SLPs, $\mathbf{L}_{t} \mathbf{D}^{T}$, represents the load in the network in correlation with the local generation. The objective of the optimization is to reduce the residual time-series by minimizing root mean square error of $\varepsilon_{t}$,

$$
\min _{\mathbf{D}, \mathbf{G}} \sqrt{\frac{1}{T_{f}} \sum_{t=1}^{T_{f}} \varepsilon_{t}^{2}}
$$

where $T_{f}$ is the length of the available time-series. The variables for the optimization algorithm, $\mathbf{D}$ and $\mathbf{G}$, are unconstrained and unbounded. The optimization algorithm is thus a linear programming problem which is solved in Python using CPLEX.

The load profile aggregated at $10 \mathrm{kV}$ is derived from measurement data in Denmark for the year 2014-15, while the available SLPs originate from a German distribution grid from the year 2016. Load profiles are decidedly dependent on weather and user behavior at the time of day. Hence, the load profile aggregated at $10 \mathrm{kV}$ and SLPs have temporal dissimilarities, which explain the presence of a residual timeseries. However, the temporal dissimilarities can be further reduced by time warping the German SLPs according to the $10 \mathrm{kV}$ aggregated load profile.

Dynamic Time Warping (DTW) was initially applied as a pattern matching algorithm wherein the time-axis fluctuation is modeled with a nonlinear time warping function by [24], [25]. The time warping function is then applied to eliminate time differences between two signals for maximum coincidence. The problem is drafted as a dynamic programming problem, with the time-warping function being the optimization variable.

DTW is used to reduce temporal mismatch between the weighted sum of SLPs and the aggregated load time-series at $10 \mathrm{kV}$ while incorporating the presence of weather-dependent generation in the $10 \mathrm{kV}-0.4 \mathrm{kV}$ network. DTW generates a time warping vector $\mathbf{F}_{t}$ given the following inputs,

$$
\mathbf{F}_{t}=d t w\left(P_{t}, \mathbf{L}_{t} \mathbf{D}^{T}-\mathbf{W}_{t} \mathbf{G}^{T}\right)
$$

The time-warping vector is then used to warp the SLP timeseries vector, $\mathbf{L}_{t}$. Let the time warped SLP vector be termed $H_{t}$,

$$
\begin{gathered}
\mathbf{H}_{t}=\mathbf{L}_{t}\left(\mathbf{F}_{\mathbf{t}}\right) \\
\mathbf{H}_{t}=\left[\begin{array}{llll}
h_{1, t} & h_{2, t} & \ldots & h_{27, t}
\end{array}\right]
\end{gathered}
$$

where $h_{i, t}, i \in \mathcal{L}$ is the load from the $i^{\text {th }}$ time warped load profile for a particular $10 \mathrm{kV}-0.4 \mathrm{kV}$ network.

Since, the optimization and dynamic time warping is performed individually for all the $10 \mathrm{kV}-0.4 \mathrm{kV}$ networks, the load time-series given by $\mathbf{H}_{t}$ is unique for every network.

\section{Profile assignment}

The optimization algorithm along with dynamic time warping provides maximum load from load time-series and installed generation capacity in a $10 \mathrm{kV}-0.4 \mathrm{kV}$ network. Next every node in the $10 \mathrm{kV}-0.4 \mathrm{kV}$ network is assigned a load profile and/or a generation profile via the profile assignment process.

Every node in the $10 \mathrm{kV}-0.4 \mathrm{kV}$ network is assigned a value for its maximum load, which is an integer multiple of the minimum load value provided as a user defined input to DiNeMo while generating the distribution network topology.

Let a $10 \mathrm{kV}-0.4 \mathrm{kV}$ network have $N$ nodes $(0.4 \mathrm{kV}$ and/or $10 \mathrm{kV}$ ), with the minimum load at any node being $s_{\text {min }}$. Maximum load at the $n^{\text {th }}$ node in the network is thus given by,

$$
x_{n}=a_{n} s_{\min } \quad \forall a_{n} \in \mathcal{Z}, n \in\{1, N\}
$$

where $a_{n}$ is an integer multiple for the $n^{\text {th }}$ node. The total maximum simultaneous load, according to DiNeMo, for a $10 \mathrm{kV}-0.4 \mathrm{kV}$ network is,

$$
X=\sum_{n=1}^{N} x_{n}=s_{\min } \sum_{n=i}^{N} a_{n}
$$


The optimization results indicate that the total maximum simultaneous load for a $10 \mathrm{kV}-0.4 \mathrm{kV}$ network is the sum of maximum loads for the load profiles $\mathbf{H}_{t}$, termed $Y$, as follows:

$$
Y=\sum_{i=1}^{27} d_{i}
$$

Let $s_{\text {min }}^{\prime}$ indicate a scaled minimum load at each node of the $10 \mathrm{kV}-0.4 \mathrm{kV}$ network, such that,

$$
\begin{gathered}
Y=s_{\text {min }}^{\prime} \sum_{n=i}^{N} a_{n}=\sum_{n=1}^{N} y_{n} \\
y_{n}=a_{n} s_{\text {min }}^{\prime} \quad \forall a_{n} \in \mathcal{Z}, n \in\{1, N\}
\end{gathered}
$$

where, $y_{n}$ is the scaled maximum load at the $n^{t h}$ node. Thus, $s_{\text {min }}^{\prime}$ can be calculated from (10) and (12) as follows,

$$
s_{\min }^{\prime}=s_{\min } \frac{Y}{X}
$$

Assume that $i^{\text {th }}$ load profile, $i \in \mathcal{L}$, is assigned to $k_{i} \in \mathcal{Z}$ nodes, in the $10 \mathrm{kV}-0.4 \mathrm{kV}$ network.

$$
N=\sum_{i=1}^{27} k_{i}
$$

Thus the maximum load for the $i^{\text {th }}$ load profile, denoted by $d_{i}$, is equally distributed amongst $k_{i}$ nodes, which implies,

$$
d_{i}=t_{i} k_{i} s_{\min }^{\prime}
$$

where, $t_{i}$ is an integer multiple assigned for $i^{\text {th }}$ load profile. Therefore $k_{i}$ can be calculated as follows,

$$
k_{i}=\operatorname{round}\left(\frac{d_{i}}{t_{i} s_{\text {min }}^{\prime}}\right)
$$

Notice that there are two unknowns in the above equation, $t_{i}$ and $k_{i}$. A heuristics based approximation is used to determine the value of $t_{i}$, based on statistical information as follows.

The SLPs from SimBench are broadly classified into 7 levels of power consumption / maximum load. A similar classification is assumed for the time warped load timeseries, $\mathbf{H}_{t}$. Let, $c_{i}$, denote the maximum load from a node which is assigned a load profile of the $i^{t h}$ power consumption level. The consumption level for each load profile imposes a condition on maximum load for each load profile which will aid is assignment of the standard load profiles to the nodes.

$$
c_{V I I} \geq c_{V I} \geq c_{V} \geq c_{I V} \geq c_{I I I} \geq c_{I I} \geq c_{I}
$$

Note that the inequality (17) is not implemented as a hard constraint. Thus, from (15) and (17) the following condition can be accepted for the order of $t_{i}$,

$$
t_{V I I} \geq t_{V I} \geq t_{V} \geq t_{I V} \geq t_{I I I} \geq t_{I I} \geq t_{I}
$$

The profile assignment starts from the nodes with maximum load according to DiNeMo by assigning a load profile with maximum power consumption level and progresses in a descending order of the maximum load. An appropriate value of $k_{i}$ is chosen in accordance with the maximum demand from the load profile, $d_{i}$ and an appropriate multiplying factor $t_{i}$.
Finally, the maximum demand for the $i^{\text {th }}$ load profile is given as follows,

$$
s_{i}=d_{i} / k_{i} \quad \forall i \in[1,27]
$$

In addition to load profiles, the aggregated PV and WPP generation capacities are assigned to appropriate nodes in the network. The following assumptions are considered while allocating the weather-dependent generation in the $10 \mathrm{kV}$ $0.4 \mathrm{kV}$ network:

- Minimum installed PV capacity: $1 \mathrm{~kW}$

- PV generation can be installed at $0.4 \mathrm{kV}$ or $10 \mathrm{kV}$ nodes

- Minimum installed WPP capacity: $300 \mathrm{~kW}$

- WPPs are installed only at $10 \mathrm{kV}$

- Installed generation at any consumer node is less than its maximum demand

Statistics for solar installations in the Danish grid, provided in [26], show that the self-consumption via PV generation is in the range of $20-30 \%$ for the residential sector and about $40 \%$ for the commercial sector, for the years in question. Residential PV plants, i.e. rooftop plants, have an installed capacity of $7 \mathrm{~kW}$ or less, in particular $3 \mathrm{~kW}$ PV installations being greatly preferred, while for the commercial sector, installed PV capacity is $7 \mathrm{~kW}$ or higher. Taking into account the statistics described in [26], the installed PV generation capacity is assumed to supply $25 \%$ of the maximum load for household loads, and $40 \%$ of the maximum load for commercial and agricultural loads.

It is assumed that the minimum installed generation for a WPP is $300 \mathrm{~kW}$ connected only at the $10 \mathrm{kV}$ nodes. In order to assign WPPs at the $10 \mathrm{kV}$ node, wind installation statistics from [23] are used as a reference. For the geographical area of the $10 \mathrm{kV}-0.4 \mathrm{kV}$ networks, the maximum onshore wind installation is in the range of $3.6 \mathrm{MW}-4 \mathrm{MW}$. However, a large number of WPPs have installed capacities of $1 \mathrm{MW}$. Hence, for assigning WPPs to the $10 \mathrm{kV}$ nodes, a maximum to minimum approach is adopted. This implies that the total installed wind power generation in a $10 \mathrm{kV} / 0.4 \mathrm{kV}$ network is divided into chunks of $3.6 \mathrm{MW}$ WPPs and a remainder value if applicable, and assigned to the $10 \mathrm{kV}$ nodes. If there are insufficient number of $10 \mathrm{kV}$ nodes, then the total installed wind generation capacity is distributed amongst the available nodes.

\section{Results}

The optimization results are depicted and described for $10 \mathrm{kV}-0.4 \mathrm{kV}$ network at Bus 27 which serves as representative network for all the $10 \mathrm{kV}-0.4 \mathrm{kV}$ networks. A power flow analysis is also performed for this network.

\section{A. Network modeling}

The optimization algorithm specifies the maximum load from the standard load profiles and installed wind and PV generation in the network as depicted in Fig.5. The X-axis in Fig.5, except for Wind and PV, indicate the names of the SLPs as described in the SimBench dataset. Briefly, $H$ represents the household SLPs, $L$ represents agricultural SLPs, $G$ represents 


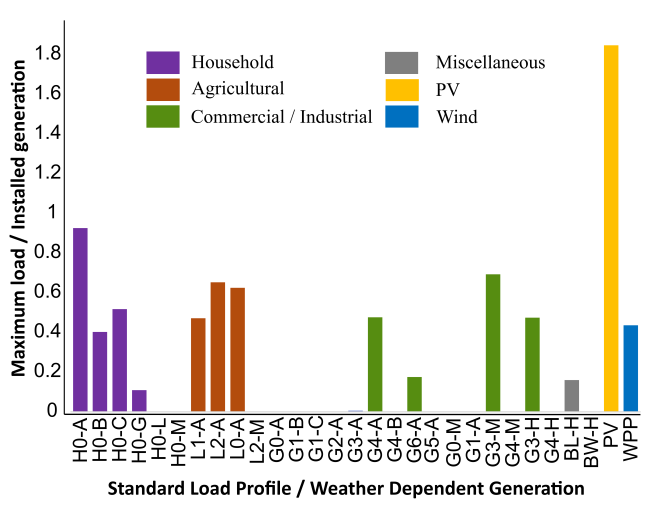

Fig. 5. Maximum demand for SLPs and total installed generation capacity for weather-dependent generation profiles

industrial/commercial slps, and the rest are miscellaneous. Note that not all the SLPs have non-zero values.

Table II presents key statistics for the loads connected to the network at Bus 27. Population density of this geographical area qualifies it to be a semi-urban/rural network, which justifies the presence of the agricultural load in equal proportion to commercial load. Note that, even though the aggregate load at $10 \mathrm{kV}$ is positive, indicating a load only network, the optimization computes $\approx 1.85 \mathrm{MW}$ of installed $\mathrm{PV}$, and $\approx 0.44 \mathrm{MW}$ of installed wind capacity which was un-observable in the aggregate load.

TABLE II

STATISTICS FOR 10 KV-0.4 KV NETWORK AT BUS 27

\begin{tabular}{|c|c|}
\hline Type of network & semi-urban/rural \\
Maximum load at Bus 27 & $3.51 \mathrm{MW}$ \\
Minimum load at Bus 27 & $0.26 \mathrm{MW}$ \\
Maximum Household load & $1.95 \mathrm{MW}$ \\
Maximum Agricultural load & $1.75 \mathrm{MW}$ \\
Maximum Commercial/Industrial load & $1.82 \mathrm{MW}$ \\
Installed PV capacity & $1.85 \mathrm{MW}$ \\
Installed WPP capacity & $0.44 \mathrm{MW}$ \\
\hline
\end{tabular}

A snapshot of the aggregated load time-series at $10 \mathrm{kV}$, weighted sum of SLPs $\left(\mathbf{L}_{t} \mathbf{D}^{T}\right)$, and the weighted sum of time-warped load profiles $\left(\mathbf{H}_{t} \mathbf{D}^{T}\right)$, is plotted in Fig. 6 for 250 time-steps. Henceforth, the aggregated load time-series at $10 \mathrm{kV}$ is named as the reference time-series. Similarly, the weighted sum of SLPs is named as the solution without DTW, and the weighted sum of time-warped load profiles is named as the solution with DTW. The root mean square error for the solution without DTW is $\approx 9 \%$, while the root mean square error for the solution with DTW is $\approx 8.1 \%$. Thus, DTW reduces the root mean square error between the aggregated load time-series at $10 \mathrm{kV}$ and the solution time-series.

It is observed from Fig. 6 that, neither the solution with DTW nor the solution without DTW replicate the aggregated load time-series completely. This implies that available SLPs, time-warped load profiles, and weather-dependent generation profiles fail to completely categorize the characteristic behavior of the aggregated load time-series. The difference between the solution with DTW and the reference time-series is the residual time-series. Another measure used to analyze the

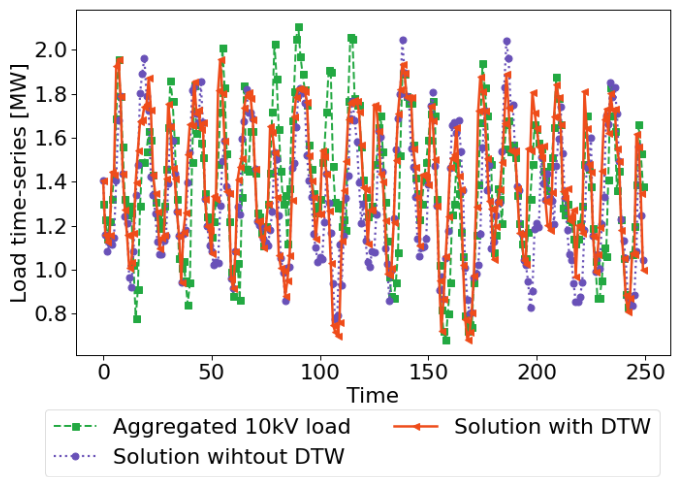

Fig. 6. Reference and solution time-series for 250 time-steps

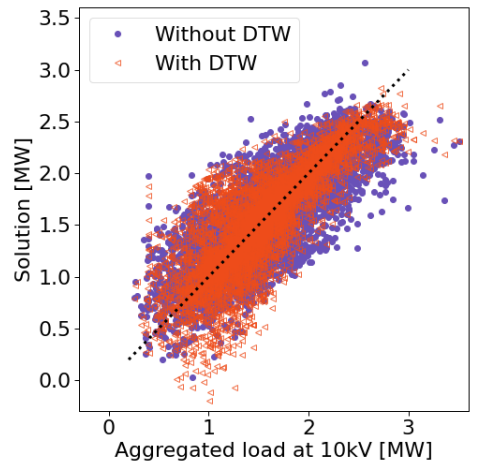

Fig. 7. Scatter plot for the solution time-series with and without DTW with respect to reference time-series

quality of the solution is the correlation between $P_{t}$ and the $\mathbf{H}_{t}$. Fig. 7 exhibits a scatter plot of the two solution profiles with and without DTW on the y-axis against the reference time-series on the $\mathrm{x}$-axis. The scatter plot suggests that the solution profile with DTW is better aligned with the reference time-series in comparison to the solution profile without DTW. This is also evident from the fact that the correlation of the solution without DTW with the reference time-series is 0.78 . DTW improves the correlation between the reference timeseries and the solution time-series to 0.83 .
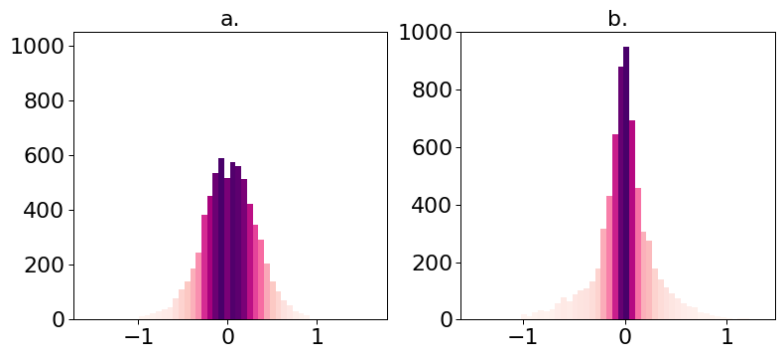

Fig. 8. a. Probability distribution for solution without DTW; b. Probability distribution for solution with DTW

The positive effect of performing DTW on the weighted sum of SLPs is most noticeable in the residual time-series. Fig. 8 captures the probability distribution of the solution without DTW and the solution with DTW. After DTW, the solution time-series is more concentrated around 0 value compared 
to the solution without DTW. The $95^{\text {th }}$ percentile value for the probability distribution of the preliminary solution is 0.523 while the $95^{t h}$ percentile for the probability distribution with DTW is 0.47 which further suggests a reduction in the mismatch between the reference and the solution.

The final step in generating the Low Voltage (LV) network, shown in Fig. 2, is to distribute all the time-warped load time-series amongst individual $400 \mathrm{~V}$ or $10 \mathrm{kV}$ nodes using the heuristic algorithm described in Section III-C.

\section{B. Power Flow}

The result of the power flow algorithm for one timestamp is provided in this section for the $10 \mathrm{kV}-0.4 \mathrm{kV}$ network at Bus 46. The power flow calculations are computed using Newton Rapson method in PyPower. Key statistics for the

TABLE III

STATISTICS FOR $10 \mathrm{KV}-0.4 \mathrm{KV}$ NETWORK AT BUS 46

\begin{tabular}{|c|c|}
\hline Type of network & urban-residential \\
Maximum load at Bus 46 & $8.0 \mathrm{MW}$ \\
Minimum load at Bus 46 & $-7.3 \mathrm{MW}$ \\
Maximum Household load & $2.8 \mathrm{MW}$ \\
Maximum Agricultural load & $0 \mathrm{MW}$ \\
Maximum Commercial/industrial load & $1.9 \mathrm{MW}$ \\
Installed PV capacity & $3.3 \mathrm{MW}$ \\
Installed WPP capacity & $36.5 \mathrm{MW}$ \\
\hline
\end{tabular}

network are tabulated in Table III. The network at Bus 46 is chosen because it contains a high share of weather-dependent renewable energy sources and it is one of the largest $10 \mathrm{kV}$ $0.4 \mathrm{kV}$ networks. A random timestamp is chosen to perform power flow to establish the plausibility of the load and generation time-series along with the $10 \mathrm{kV}-0.4 \mathrm{kV}$ networks. All the $10 \mathrm{kV} / 400 \mathrm{~V}$ transformers are assumed to be equipped with off-load tap-changers and $60 \mathrm{kV} / 10 \mathrm{kV}$ transformers are equipped with on-load tap-changers, with taps set to nominal position.

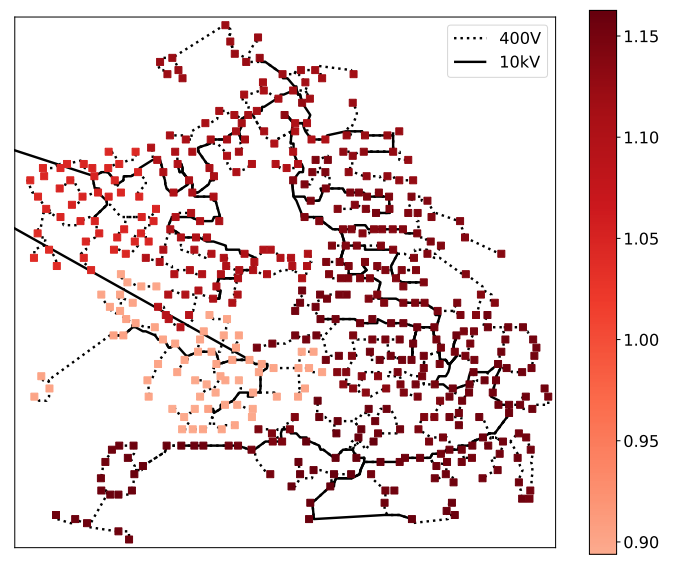

Fig. 9. Voltages at all nodes for one timestamp in the $10 \mathrm{kV}-0.4 \mathrm{kV}$ network connected at Bus 46 - Load: $31 \mathrm{MW}$ - Generation: 32.62 MW - Line Losses: $11 \mathrm{MW}$
The required load from the network at the $60 \mathrm{kV}-10 \mathrm{kV}$ substation is $31 \mathrm{MW}$. The total generation from the PV and WPPs installed in the network is $32.62 \mathrm{MW}$. The total active power line loss in the network is $11 \mathrm{MW}$. Fig. 9 depicts the voltages at all the nodes in the $10 \mathrm{kV}-0.4 \mathrm{kV}$ network for this timestamp. Note that the nodes at the end of the distribution line experience higher voltages than the nodes close to the $60 \mathrm{kV}-10 \mathrm{kV}$ substation. This is due to a high amount of distributed generation in the network.

\section{Summary}

This paper proposes and presents the methodology used for the development of a multi-voltage active distribution network model, named DTU 7k-Bus Active Distribution Network (DTU-ADN). The DTU-ADN spans across 3 voltage levels, $60 \mathrm{kV}$ - $10 \mathrm{kV}-0.4 \mathrm{kV}$, and hosts an abundant amount of distributed renewable energy sources, primarily wind and solar power, connected across all the voltage levels. The distribution network model is developed using a top-down approach, premised on the $60 \mathrm{kV}$ distribution network model and correlations between load demand and wind and/or solar generation in a low voltage distribution network. The approach presented in this research expands the initial $60 \mathrm{kV}$ network with $1410 \mathrm{kV}-0.4 \mathrm{kV}$ networks at different $60 \mathrm{kV}-10 \mathrm{kV}$ substation nodes. Aggregated load (active and reactive power) and generation time-series, for a period of about 10 months, accompanying the $60 \mathrm{kV}$ network, is used to assign load and generation profiles to the $10 \mathrm{kV}-0.4 \mathrm{kV}$ nodes.

The $60 \mathrm{kV}$ network topology represents a real distribution network in Denmark while the $10 \mathrm{kV}-0.4 \mathrm{kV}$ networks are simulated from geographical data with the Distribution Network Modeling (DiNeMo) tool [19]. Measurement data is used to derive the aggregated load time-series at $60 \mathrm{kV}$ nodes. The load profiles assigned to $10 \mathrm{kV}$ and $0.4 \mathrm{kV}$ nodes represent commercial/industrial, agricultural, household and miscellaneous loads. Finally, meteorological data is used to simulate wind and solar generation time-series using CorRES [21].

An optimization problem is formulated to calculate the aggregate proportion of the 27 standard load profiles along with wind and solar generation in one $10 \mathrm{kV}-0.4 \mathrm{kV}$ network. The objective of the optimization is to minimize the difference between the aggregated load profile at $60 \mathrm{kV}-10 \mathrm{kV}$ substation, and summation of the 27 standard load profiles with wind and solar generation. The optimization routine emerges with a preliminary result of a $10-20 \%$ difference between the aforementioned load profiles and a correlation of 0.6-0.9. Further on, the dynamic time warping method reduces the difference up to $5-15 \%$ with a correlation of $0.8-0.9$. The difference in the dynamic time-warped time-series and the aggregated time series at $60 \mathrm{kV}-10 \mathrm{kV}$ substation is termed as the residual time-series and is also provided with the DTUADN data.

The aggregated $60 \mathrm{kV}$ time-series data is derived from measurement data in Denmark, whereas, the standard load profiles from [20] originate from Germany. In addition, the load time series are also measured in different meteorological 
years, namely 2014-15 and 2016. A load time series represents the consumption pattern of consumers connected to the given network and is deeply subject to changes in the weather and temperature. This is one of the major intractable sources of difference between the optimized load profile and the aggregated $60 \mathrm{kV}$ load profile. Although the wind and solar generation time series is simulated from meteorological data from the same geographical location as the $10 \mathrm{kV}-0.4 \mathrm{kV}$ networks, it does not consider expected or unexpected shutdowns or ramping down of the wind and solar power plants. Such shut-down or ramping down of the generated power may arise due to maintenance period or failure of the power plants, storm-shut downs, strategic ramp downs, etc. However, the aggregated load profile at the $60 \mathrm{kV}-10 \mathrm{kV}$ substation implicitly incorporates this information as it is derived from measurement data. Failure to account for the expected and unexpected decrease in generation adds another intractable source of difference in the optimization.

The 17 networks at $10 \mathrm{kV}-0.4 \mathrm{kV}$ epitomize different characteristic medium-low voltage distribution networks via different proportions of commercial, household, and agricultural profiles. Since the network topology are simulated from geographical data, they represent diversity in terms of the network topology, energy density, and can be grouped into urban, semiurban, and rural networks. Wind and solar power produce from $2 \%$ to more than $300 \%$ of the total energy demand in different $10 \mathrm{kV}-0.4 \mathrm{kV}$ networks for the given period. Thus, the DTUADN houses $10 \mathrm{kV}-0.4 \mathrm{kV}$ networks with varying amounts of renewable energy penetrations.

The DTU-ADN provides an opportunity to study multivoltage distribution networks with distinct characteristics and RES penetration levels. On one hand, the distribution networks can be employed to study, control, and optimize voltage profiles, line losses, network asset operation with high RES penetration. While on the other hand, the multi-voltage models can be used to promote active participation of distribution networks in the provision of flexibility services, through controllable power electronics-based distributed generation. The DTU-ADN also provides the adaptability to incorporate additional assets such as storage, electric vehicle charging, or analyze demand response in the distribution network.

\section{ACKNOWLEDGMENT}

This project has received funding from the European Union's Horizon 2020 research and innovation program under the Marie Sklodowska-Curie grant agreement No 861398 and Energy Technology Development and Demonstration Program (EUDP) 2019-II IEA Task 41 Journal Number 64019-0518. PSfuture project (La Cour Fellowship, DTU Wind Energy) is also acknowledged for support during this project.

\section{REFERENCES}

[1] R. A. Walling, R. Saint, R. C. Dugan, J. Burke, and L. A. Kojovic, "Summary of distributed resources impact on power delivery systems," IEEE Transactions on Power Delivery, vol. 23, no. 3, pp. 1636-1644, 2008.

[2] K. A. Alboaouh and S. Mohagheghi, "Impact of Rooftop Photovoltaics on the Distribution System," Journal of Renewable Energy, vol. 2020, pp. 1-23, 2020.
[3] A. Baviskar, A. D. Hansen, K. Das, and M. Koivisto, "Challenges of Future Distribution Systems with a Large Share of Variable Renewable Energy Sources - Review," 19th Wind Integration Workshop 2020, no. November, 2020.

[4] K. Strunz, "Developing Benchmark Models," Symposium A Quarterly Journal In Modern Foreign Literatures, vol. 6, no. Coll, pp. 4-5, 2005.

[5] K. Rudion, A. Orths, Z. A. Styczynski, and K. Strunz, "Design of benchmark of medium voltage distribution network for investigation of DG integration," 2006 IEEE Power Engineering Society General Meeting, PES, p. 6 pp., 2006.

[6] K. Strunz, C. Abbey, C. Andrieu, R. C. Campbell, and R. Fletcher, Benchmark Systems for Network Integration of Renewable and Distributed Energy Resources, 2009, no. July.

[7] K. P. Schneider, B. A. Mather, B. C. Pal, C. W. Ten, G. J. Shirek, H. Zhu, J. C. Fuller, J. L. Pereira, L. F. Ochoa, L. R. De Araujo, R. C. Dugan, S. Matthias, S. Paudyal, T. E. McDermott, and W. Kersting, "Analytic Considerations and Design Basis for the IEEE Distribution Test Feeders," IEEE Transactions on Power Systems, vol. 33, no. 3, pp. 3181-3188, 2018.

[8] D. P. Chassin, K. Schneider, and C. Gerkensmeyer, "GridLAB-D: An open-source power systems modeling and simulation environment," Transmission and Distribution Exposition Conference: 2008 IEEE PES Powering Toward the Future, PIMS 2008, pp. 1-5, 2008.

[9] J. Amme, G. Pleßmann, J. Bühler, L. Hülk, E. Kötter, and P. Schwaegerl, "The eGo grid model: An open-source and open-data based synthetic medium-voltage grid model for distribution power supply systems," Journal of Physics: Conference Series, vol. 977, no. 1, 2018.

[10] M. Sarstedt, G. Steffen, C. Blaufus, and L. Hofmann, "Modelling of Integrated Transmission and distribution grids based on synthetic distribution grid models," IEEE Milan Power, 2019.

[11] C. Matke, W. Medjroubi, and D. Kleinhans, "SciGRID - An Open Source Reference Model for the European Transmission Network (v0.2)," no. November, 2016. [Online]. Available: http://www.scigrid.de

[12] D. Montenegro, M. Hernandez, and G. A. Ramos, "Real time OpenDSS framework for distribution systems simulation and analysis," Proceedings of the 2012 6th IEEE/PES Transmission and Distribution: Latin America Conference and Exposition, T and D-LA 2012, pp. 5-9, 2012.

[13] S. Meinecke, A. Klettke, J. Dickert, M. Hable, F. Fischer, M. Braun, A. Moser, and C. Rehtanz, "General planning and operational principles in german distribution systems used for Simbench," no. June, pp. 3-6, 2019. [Online]. Available: www.simbench.net

[14] S. Coast, "Open Street Maps," 2004. [Online]. Available: www. openstreetmap.org/

[15] D. Sarajlić and C. Rehtanz, "Low Voltage Benchmark Distribution Network Models Based on Publicly Available Data," Proceedings of 2019 IEEE PES Innovative Smart Grid Technologies Europe, ISGTEurope 2019, pp. 1-5, 2019.

[16] S. Meinecke, N. Bornhorst, and M. Braun, "Power System Benchmark Generation Methodology," no. February, 2019.

[17] A. Navarro-Espinosa and L. Ochoa, "Dissemination Document " Low Voltage Networks Models and Low Carbon Technology Profiles "," Ph.D. thesis The University of Manchester, vol. 44, no. June, pp. 127, 2015.

[18] A. Baviskar, A. D. Hansen, K. Das, and M. Koivisto, "DTU 7k-Bus Active Distribution Network," 2021. [Online]. Available: https://doi.org/10.11583/DTU.c.5389910.v1

[19] M. Grzanic, M. G. Flammini, and G. Prettico, "Distribution network model platform: A first case study," Energies, vol. 12, no. 21, 2019.

[20] C. Spalthoff, D. Sarajlić, C. Kittl, S. Drauz, T. Kneiske, C. Rehtanz, and M. Braun, "SimBench : Open source time series of power load, storage and genera- tion for the simulation of electrical distribution grids," no. September, pp. 447-452, 2019.

[21] M. Koivisto, K. Das, F. Guo, P. Sørensen, E. Nuño, N. Cutululis, and P. Maule, "Using time series simulation tools for assessing the effects of variable renewable energy generation on power and energy systems," Wiley Interdisciplinary Reviews: Energy and Environment, vol. 8, no. 3, pp. 1-15, 2019.

[22] ESMAP, SOLARGIS, WB, and IFC, "Global Solar Atlas," p. 1, 2019. [Online]. Available: https://globalsolaratlas.info/?m=sg:ghi

[23] R. T. Sataloff, M. M. Johns, and K. M. Kost, "Energistyrelsen." [Online]. Available: https://ens.dk/

[24] H. Sakoe and S. Chiba, "Dynamic Programming Algorithm Optimization for Spoken Word Recognition," IEEE Transactions on Acoustics, Speech, and Signal Processing, vol. 26, no. 1, pp. 43-49, 1978.

[25] L. Rabiner and B.-H. Juang, Fundamentals of speech recognition, 1993.

[26] A. Peter;" "National Survey Report of PV Power Applications in Denmark," PA Energy Ltd. Denmark, Tech. Rep. August, 2016. 